\title{
Heterogeneously integrated III-V-on-Si microring resonators: a building block for programmable photonic integrated circuits
}

\author{
Jiayang Li $\odot$, Bo Xue Tan॰, Mingle Tian, and Andrew W. Poon $\odot *$ \\ The Hong Kong University of Science and Technology, Photonic Device Laboratory, \\ Department of Electronic and Computer Engineering, Clear Water Bay, Kowloon, \\ Hong Kong SAR, China
}

\begin{abstract}
Beyond various application-specific photonic integrated circuits (PICs), a programmable PIC comprising identically designed generic building blocks with multiple active functionalities under different operational conditions is an attractive design option. Here, we propose and demonstrate proof-of-concept experiments of a heterogeneously integrated III-V-on-Si microring resonator (MRR) as such a versatile building block. The MRR can function as an electro-optical channel add-drop switch with signal amplification, a directly modulated injection laser, or a microcavity photodetector under different electrical bias conditions. We discuss the fabrication process for our heterogeneous integration platform based on an atomic layer deposition-assisted molecular bonding technique. (C) The Authors. Published by SPIE under a Creative Commons Attribution 4.0 Unported License. Distribution or reproduction of this work in whole or in part requires full attribution of the original publication, including its DOI. [DOI: 10.1117/1.JOM.1.3 $.034002]$
\end{abstract}

Keywords: photonic integrated circuit; programmable photonics; heterogeneous integration; microring resonator; optical switch; laser.

Paper 20022 received Dec. 19, 2020; accepted for publication Apr. 28, 2021; published online May 14, 2021.

\section{Introduction}

Photonic integrated circuits (PICs) based on silicon photonics have been intensely developed over the past two decades and recently commercialized. A wide range of application-specific PICs have been demonstrated for various purposes including optical communications and datacenter interconnections, biosensing, quantum optics, and optical neuromorphic computing. ${ }^{1,2} \mathrm{By}$ heterogeneously integrating III-V compound semiconductors as a gain or active medium onto Si photonics through wafer-bonding techniques, ${ }^{3-5}$ researchers have integrated on silicon PICs electro-optic (EO) modulators, ${ }^{6-8}$ switches, ${ }^{9,10}$ injection lasers, ${ }^{11,12}$ and semiconductor optical amplifiers (SOAs), ${ }^{13,14}$ thus enabling more functional application-specific PICs including optical transceivers ${ }^{15}$ and on-chip two-dimensional beam-steering for light detection and ranging. ${ }^{16}$

Application-specific PICs comprising tailor-designed components aim at optimal performance for the targeted applications, e.g., energy efficiency, footprint, and switching speed, for an optical switch network. They generally require optimum design parameters for each component. Moreover, the PIC network arrangement is generally fixed after fabrication. Recently, emerging applications such as neuromorphic photonic computing for artificial intelligence require a larger flexibility for reconfiguration of on-chip arrangements. ${ }^{17}$

We believe that a programmable PIC comprising identically designed generic building blocks with multiple active functionalities under different operational conditions is an attractive design option to provide flexibility for reconfiguration of on-chip arrangements for future applications. This is analogous to the electronic field-programmable gate array (FPGA). Recently, researchers have reported several theoretical works describing the principles and the PIC configurations based on identically designed beam splitters or integrated Mach-Zehnder interferometers (MZIs).$^{18}$ Programmable photonic arrays with MZIs meshed in various configurations featuring

*Address all correspondence to Andrew W. Poon, eeawpoon@ust.hk 
multiple functionalities have been experimentally demonstrated. ${ }^{19,20}$ However, these arrays are limited to all-silicon thermal-optic (TO) tuned MZIs, which feature a limited function of beam splitting, a device length of $\sim 1 \mathrm{~mm}$, and a large power consumption of a few tens of $\mathrm{mW}$ for thermal tuning. ${ }^{21}$ Moreover, silicon MZIs do not offer the necessary resonance optical feedback for lasing actions even if integrated with an III-V gain medium.

A heterogeneously integrated III-V-on-Si microring resonator (MRR) can serve as a suitable building block. By applying an electrical bias across the III-V active layer, we can switch the MRR operation from a reverse-biased microcavity photodetector (PD) for on-chip channelselective data readout to a forward-biased wavelength-channel-agile EO add-drop switch with loss compensation, to a directly modulated injection laser as an on-chip source, all using the same (programmable) device structure. Figure 1(a) shows schematically the three different operational regimes with different electrical bias conditions. Figure 1(b) shows schematically the resonance transmission spectra of the III-V-on-Si MRR add-drop filter at the drop- and through-ports, without injection and with injection beyond the transparency.

At a low injection current below the transparency or upon a reverse bias, the MRR resonances in the 1550-nm wavelengths are only weakly coupled by the bus-waveguide due to the absorption of the III-V medium. The injection toward the transparency turns the absorbing medium into a gain medium. The reduced absorption loss enhances the coupling to the cavity resonances, thus switching the transmission from the through-port to the drop-port. The data transmission will be amplified with an injection slightly beyond the transparency.

The cavity resonance wavelength upon injection depends on (i) the free-carrier dispersion (FCD) (blueshift) of the III-V layers across the diode, (ii) the band-filling effect of the multiplequantum-well (MQW) in the III-V layers (blueshifting the gain spectrum), ${ }^{22}$ and (iii) the currentinduced TO effect (redshift) inside the III-V-on-Si microcavity. We note that it is desirable to operate the MRR as an EO switch, with a response time on the order of $\sim \mathrm{ns},{ }^{7,9}$ at a low injection level before the TO effect saturates the resonance wavelength blueshift.

Figure 1(c) shows schematically a cascaded cross-grid network in which the III-V-on-Si MRRs operate differently with various programmed bias voltages to function separately as a directly modulated laser source, an EO switch, and a microcavity PD, all programmed by an FPGA. However, the bandwidth of the cascaded network will inevitably suffer; thus one should avoid excessive routing in the network to ensure a reasonable data bandwidth and avoid excessive noise. The readout photocurrent is first converted into a voltage form and amplified through external electronic circuits. The inputs' analog-to-digital conversion is realized by the built-in analog-to-digital converter (ADC), while the outputs' digital-to-analog conversion is realized by an external digital-to-analog converter (DAC). The external electronic circuits are connected with our photonic circuits through a printed circuit board.

In this paper, we show as a proof-of-concept the design, fabrication, and characterization of our III-V-on-Si MRRs operated under different bias voltages. Figure 1(d) shows schematically the structure of a heterogeneously integrated III-V-on-Si MRR, with an InGaAs/InGaAsP MQW serving as the gain section. We realize the MRR using an atomic layer deposition (ALD)-assisted

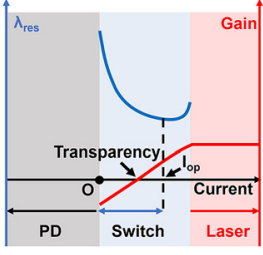

(a)

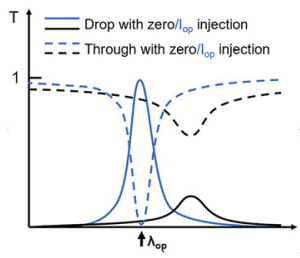

(b)

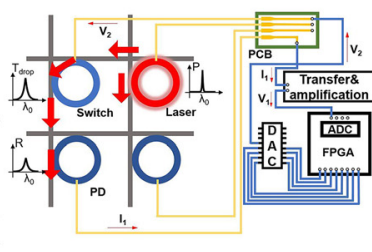

(c)

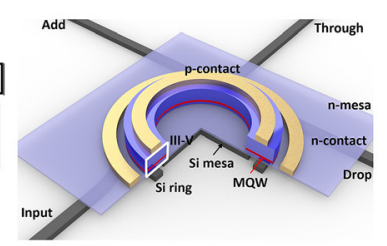

(d)

Fig. 1 Schematics of (a) the MRR round-trip gain and resonance wavelength $\left(\lambda_{\text {res }}\right)$ in different operational regimes with different bias conditions ( $I_{\mathrm{op}}$ : operating current beyond the transparency); (b) the corresponding resonance transmissions at the through- and drop-ports with zero or $I_{\text {op }}$ injection; (c) a programmable PIC comprising identically designed MRRs under different operational regimes feedback-controlled by an FPGA (P: output power, T: transmission, R: responsivity); and (d) the structure of a heterogeneously integrated III-V-on-Si MRR coupled to a silicon waveguide crossing as an add-drop filter/a switch/a laser/a PD. 
molecular bonding. We note that there are other heterogeneous integration methods such as adhesive polymer bonding, ${ }^{3}$ silica molecular bonding, ${ }^{23}$ and hetero-epitaxy. ${ }^{24}$

\section{Design and Fabrication}

Figures 2(a) and 2(b) show schematically the top- and cross-sectional views of the III-V-on-Si MRR add-drop filter/switch, respectively. We couple the silicon MRR to two 90 deg-curved buswaveguides joined with a multimode-interference (MMI) waveguide crossing in an add-drop configuration. The Si MMI waveguide crossing enables individual MRRs to be cascaded in a cross-grid network. The Si layer thickness is $340 \mathrm{~nm}$, with a rib waveguide etched height of $140 \mathrm{~nm}$ and a microring width of $1.5 \mu \mathrm{m}$. The silicon rib waveguide design minimizes the exposure of the mode to the etched silicon sidewalls for a lower scattering loss while allowing a silicon slab layer to conduct away the heat generated from the III-V active region.

We use commercially available III-V MQW 2" wafers with six InGaAs/InGaAsP quantum wells to provide gain centered at around $1530 \mathrm{~nm}$ (measured by photoluminescent experiment) for transverse electric (TE)-polarized modes. We design a $5 \mu \mathrm{m}$-wide III-V microring to ensure

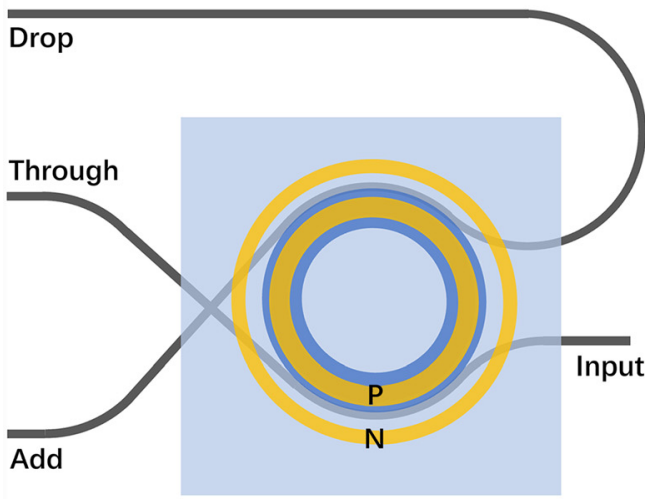

(a)

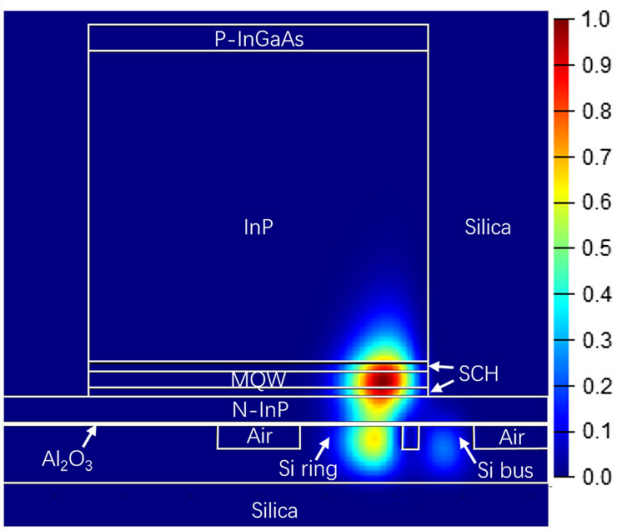

(c)

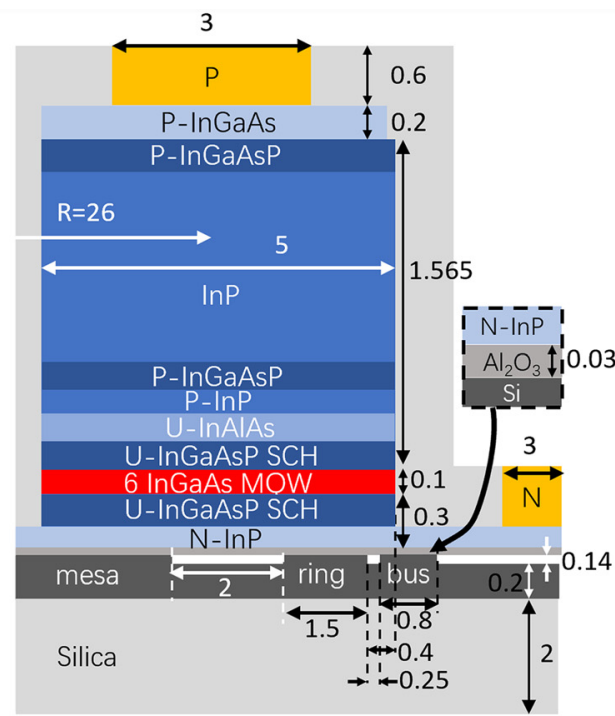

(b)

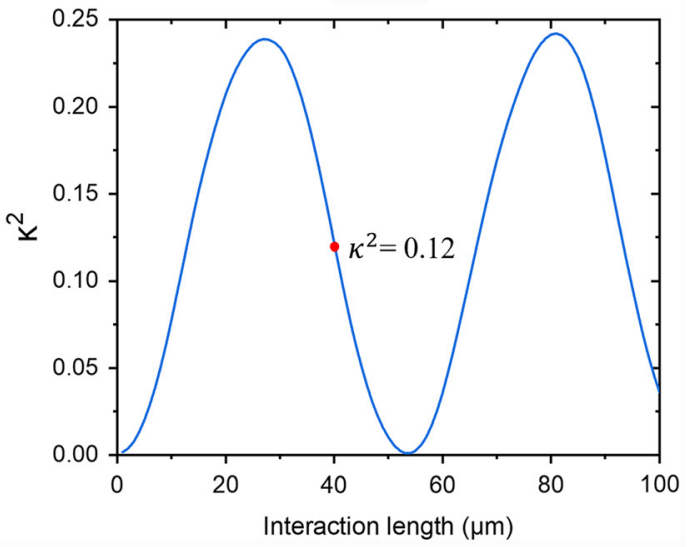

(d)

Fig. 2 Schematics of the III-V-on-Si MRR (a) top-view and (b) cross-sectional views. The units are in $\mu \mathrm{m}$. SCH: separate confinement heterostructure. Inset: zoom-in view of the $\mathrm{Al}_{2} \mathrm{O}_{3}$ bonding layer. (c) Numerically simulated hybrid mode in the III-V-on-Si MRR and in proximity to the buswaveguide coupling region. (d) Numerically simulated power coupling coefficient $\kappa^{2}$ as a function of interaction length between the MRR and the bus-waveguide. 
a sufficiently wide waveguide for a reliable lift-off of the ring-shaped $3 \mu \mathrm{m}$-wide p-electrode on top the waveguide.

We design a $26 \mu$ m-radius III-V microring. The III-V microring outer sidewall extends by $400 \mathrm{~nm}$ beyond the Si microring outer sidewall to constrain the hybrid mode distribution radially to the silicon microring and away from the III-V microring sidewall for a lower sidewall scattering loss. We position the n-electrode on the InP slab outside the microring so that the injected carriers travel radially near the III-V microring rim region for a good spatial overlap with the optical mode in the III-V microring.

Figure 2(c) shows the numerically simulated electric-field amplitude distribution of the TE hybrid mode in the coupling region between the III-V-on-Si MRR structure and the buswaveguide. Within the MRR, the mode exhibits a power spatial overlap of $19 \%$ with the MQW region and an effective refractive index of $\sim 3.43$. This is close to the effective refractive index of $\sim 3.40$ of the $0.8 \mu \mathrm{m}$-wide silicon bent bus-waveguide, which is wrapped along an arc angle of $90 \mathrm{deg}$ of the silicon microring. We design a $250-\mathrm{nm}$ coupling gap spacing between the silicon microring and the bus-waveguide. The III-V microring outer sidewall thus spatially overlaps with the silicon bus-waveguide. Our numerical simulations of the designed structure suggest an energy coupling coefficient $\kappa^{2} \sim 12 \%$ between the III-V-on-Si MRR and the Si buswaveguide, with a coupling length of $40 \mu \mathrm{m}$, as shown in Fig. 2(d).

We also design a single-bus-waveguide-coupled III-V-on-Si MRR as a test structure, with the same III-V waveguide cross-section. Here, we adopt the silicon microring width, the silicon buswaveguide width, and the coupling gap spacing to be $1 \mu \mathrm{m}, 800$, and $200 \mathrm{~nm}$, respectively. The III-V waveguide sidewall extends by $300 \mathrm{~nm}$ beyond the silicon microring in this test structure. We numerically calculate the effective refractive index of the vertically coupled mode of the III-V microring and the silicon microring to be $\sim 3.43$, which is close to the effective refractive index of the silicon bus-waveguide of $\sim 3.40$. The simulation result (not shown) suggests an energy coupling coefficient $\kappa^{2} \sim 16 \%$ between the III-V-on-Si MRR and the Si bus-waveguide, with a coupling length of $40 \mu \mathrm{m}$. Table 1 summarizes the relevant device parameters for both the

Table 1 Device parameters for our III-V-on-Si MRR devices.

\begin{tabular}{|c|c|c|}
\hline & Description & Add-drop (test, single bus) \\
\hline \multirow[t]{4}{*}{ III-V gain medium layer } & III-V outer radius $\left(R_{I I I-V}\right)$ & $28.5 \mu \mathrm{m}$ \\
\hline & III-V waveguide width $\left(W_{I I I-V}\right)$ & $5 \mu \mathrm{m}$ \\
\hline & III-V ring area $\left(A_{I I I-V}\right)$ & $817 \mu \mathrm{m}^{2}$ \\
\hline & Number of QWs & 6 \\
\hline \multirow[t]{5}{*}{ Silicon passive layer } & Bonding interlayer thickness & $32 \mathrm{~nm}$ \\
\hline & Silicon core thickness $\left(T_{s i}\right)$ & $340 \mathrm{~nm}$ \\
\hline & Silicon slab thickness ( $\left.T_{\text {slab }}\right)$ & $200 \mathrm{~nm}$ \\
\hline & Silicon ring waveguide width $\left(W_{s i}\right)$ & $1.5 \mu \mathrm{m}(1 \mu \mathrm{m})$ \\
\hline & Silicon cavity radius $\left(R_{s i}\right)$ & $28.1 \mu \mathrm{m}(28.2 \mu \mathrm{m})$ \\
\hline \multirow[t]{3}{*}{ Interaction region } & Interaction length $\left(L_{\text {int }}\right)$ & $40 \mu \mathrm{m}$ \\
\hline & Silicon bus-waveguide width ( $W_{\text {bus }}$ ) & $800 \mathrm{~nm}$ \\
\hline & Coupling gap $\left(W_{\text {gap }}\right)$ & $250 \mathrm{~nm}(200 \mathrm{~nm})$ \\
\hline \multirow[t]{3}{*}{ Electrodes } & Electrode width & $3 \mu \mathrm{m}$ \\
\hline & P-electrode metal & $\mathrm{Ti} / \mathrm{Pt} / \mathrm{Au}$ \\
\hline & $\mathrm{N}$-electrode metal & $\mathrm{Ge} / \mathrm{Au} / \mathrm{Ni} / \mathrm{Au}$ \\
\hline
\end{tabular}




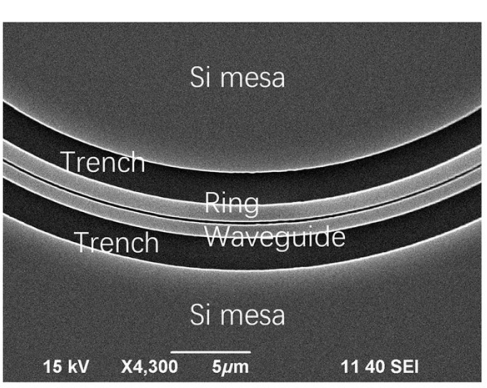

(a)

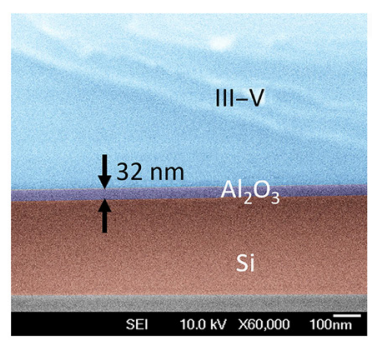

(c)

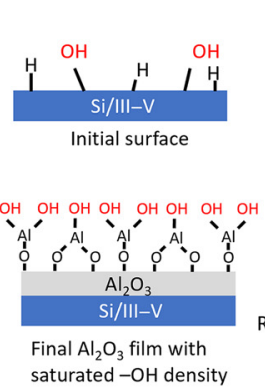

saturated $-\mathrm{OH}$ density

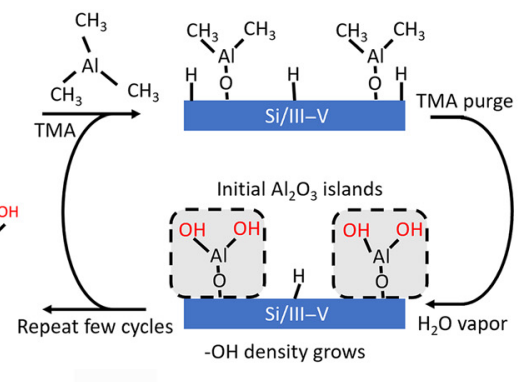

(b)

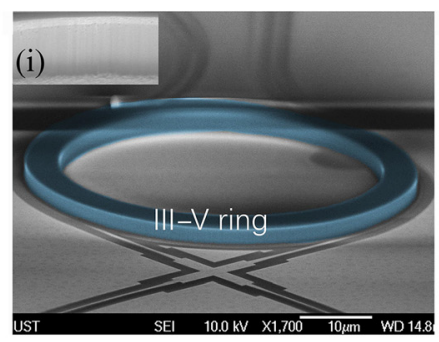

(d)

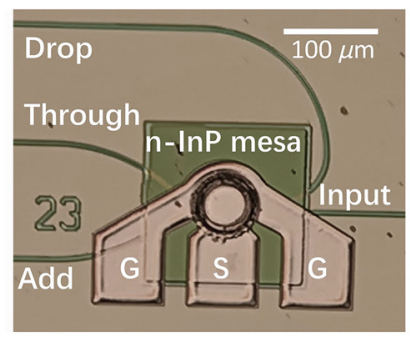

(e)

Fig. 3 (a) SEM of the fabricated Si microring-waveguide pattern after DRIE. (b) Schematic illustration of the principle of the ALD-assisted molecular bonding. (c) SEM of the bonding interface. (d) SEM of the III-V ring waveguide on the Si ring after ICP-RIE. Inset (i): a smooth III-V ring sidewall. (e) Optical microscope image of a device after all electrical depositions.

MRR add-drop filter configuration and the MRR coupled with a single bus-waveguide test structure.

We define the Si device patterns using electron-beam lithography (JEOL 6300). We adopt $2-\mu \mathrm{m}$-wide air trenches to separate the device patterns from the silicon mesa to retain most of the Si film to mechanically support the wafer-bonded III-V layer. We pattern the Si layer using $\mathrm{SF}_{6}$-based deep-reactive-ion-etching (DRIE). Figure 3(a) shows the scanning electron micrograph (SEM) of the fabricated Si device pattern in the vicinity of the silicon ring with the coupled waveguide.

We adopt the ALD-assisted molecular bonding to bond the MQW wafer onto the patterned $\mathrm{Si}$ sample. Figure 3(b) shows the process schematics. We alternate between trimethyl-aluminum (TMA, $\mathrm{Al}\left(\mathrm{CH}_{3}\right)_{3}$ ) and $\mathrm{H}_{2} \mathrm{O}$ vapor pulse gas precursors in our ALD process to attain various ultra-uniform films with highly controllable thicknesses. ${ }^{25}$

The TMA first reacts with the pre-existing sparse hydroxyl groups $(-\mathrm{OHs})$ on the silicon and III-V surfaces. Then, we purge the TMA and supply $\mathrm{H}_{2} \mathrm{O}$ vapor to replace the two $-\mathrm{CH}_{3}$ groups with two $-\mathrm{OH}$ groups to complete one cycle. Through this process, the $-\mathrm{OH}$ density on the bonding surfaces increases and the $\mathrm{Al}_{2} \mathrm{O}_{3}$ islands grow by cycles. It has been reported that $\sim 40$ cycles are required to merge all islands and to form a very compact $\mathrm{Al}_{2} \mathrm{O}_{3}$ film. ${ }^{26}$ Afterward, every $\mathrm{H}_{2} \mathrm{O}$ vapor process cycle in principle creates a surface with an $-\mathrm{OH}$ on every surface site. This ALD process could generate an - $\mathrm{OH}$ density of 4.5 times higher than the plasma-assisted process $^{27}$ with a simpler process flow as only one dry chamber is involved.

We deposit $\sim 15 \mathrm{~nm}$ of $\mathrm{Al}_{2} \mathrm{O}_{3}$ at $300^{\circ} \mathrm{C}$ on both $\mathrm{Si}$ and MQW samples using an Oxford ALD system. We mate the two samples at $300^{\circ} \mathrm{C}$ under a pressure of $2 \mathrm{MPa}$ in a vacuum ambient. The $-\mathrm{OHs}$ on both surfaces react and create permanent covalent $-\mathrm{O}-$ bonds. We obtain a large bonding yield ( $>80 \%$ area of the III-V film remains bonded after substrate removal). Figure 3(c) shows the thin $(\sim 32 \mathrm{~nm}) \mathrm{Al}_{2} \mathrm{O}_{3}$ bonding layer.

We use $\mathrm{HCl}: \mathrm{H}_{2} \mathrm{O}(4: 1)$ and $\mathrm{H}_{2} \mathrm{SO}_{4}: \mathrm{H}_{2} \mathrm{O}_{2}: \mathrm{H}_{2} \mathrm{O}(1: 1: 10)$ solutions to remove the InP substrate and the etch stop layers to expose the III-V device layer. We deposit a layer of plasmaenchanced chemical vapor depostion (PECVD) silicon nitride ( $\mathrm{SiN}$ ) as a hardmask for etching the III-V layer. We adopt electron-beam lithography for patterning the III-V layer to ensure a misalignment of $<50 \mathrm{~nm}$ between the III-V and Si patterns. We etch the III-V microring using 
Li et al.: Heterogeneously integrated III-V-on-Si microring resonators: a building block...

an $\mathrm{BCl}_{3}$-based inductively coupled plasma RIE (ICP-RIE) process. We stop the ICP-RIE within the MQW region and remove the remaining InGaAsP with a diluted $\mathrm{H}_{2} \mathrm{SO}_{4}: \mathrm{H}_{2} \mathrm{O}_{2}: \mathrm{H}_{2} \mathrm{O}$ (1:1:10) solution to ensure a smooth n-InP layer surface for electrode deposition. Figure 3(d) shows the etched III-V microring with a smooth sidewall on top of the Si microring add-drop switch structure.

We pattern the n-InP mesas using a $\mathrm{HCl}: \mathrm{H}_{2} \mathrm{O}(1: 1)$ solution to electrically isolate different devices. We adopt a standard recipe for the deposition of the $n-(\mathrm{Ge} / \mathrm{Au} / \mathrm{Ni} / \mathrm{Au})$ and p-electrical $(\mathrm{Ti} / \mathrm{Pt} / \mathrm{Au})$ contacts. We deposit a 500-nm $\mathrm{PECVD} \mathrm{SiO}_{2}$ as an upper-cladding layer and open the via-holes using the DRIE process. Finally, we deposit a copper film and etch it to form the probe metal pads. Figure 3(e) shows the optical micrograph of the fabricated device.

\section{Characterization of the Device Functionalities with Different Operational Regimes}

We cleave the fabricated chip and glue it on a thermal-electric controller (TEC). Figure 4(a) shows schematically the experimental setup for characterization of the fabricated devices. We use a wavelength-tunable continuous-wave (CW) laser light source for the transmission experiments. We align the laser light in TE polarization using a polarization controller. We end-fire the light into the chip using a lensed single-mode fiber (SMF) and collect the out-coupled light using another lensed SMF. We set the bias voltages across the devices using a radio-frequency probe and read out their electrical responses using a source meter. We use a high-speed bias-tee to combine the direct-current (DC) bias with the alternating-current modulation signal from a pattern generator. We image the chip from the top using a longworking-distance objective lens onto either a near-infrared (NIR) camera or a charge-coupleddevice camera. We measure the optical and electrical responses of the MRR devices under various bias conditions using the optical power meter, the PD connected to the oscilloscope, and the optical spectrum analyzer (OSA).

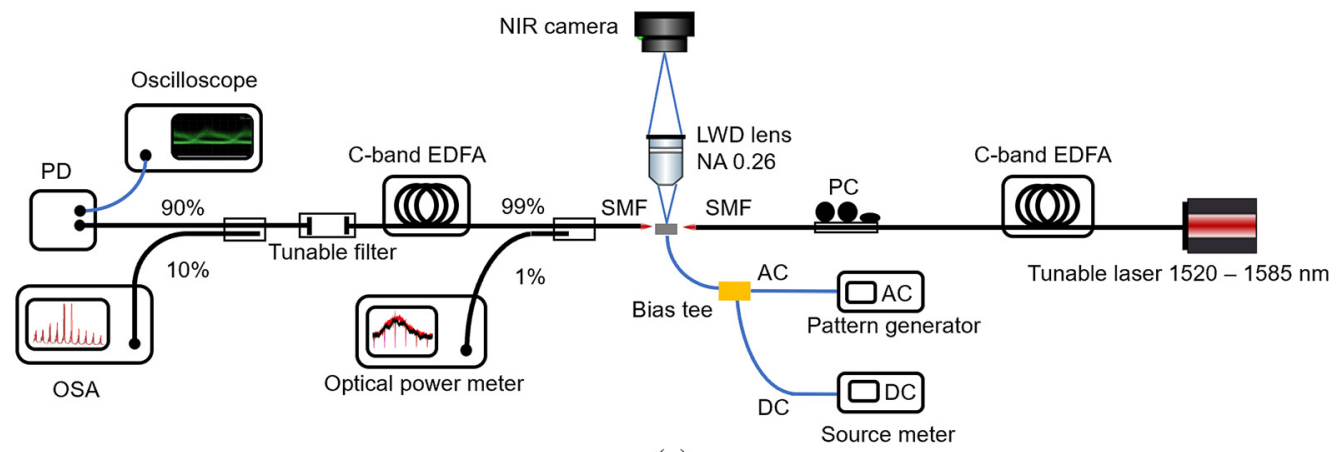

(a)

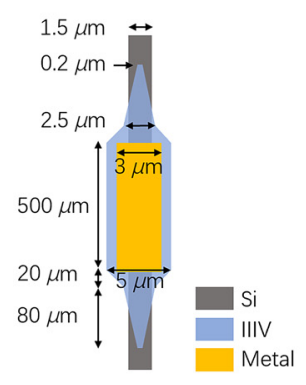

(b)

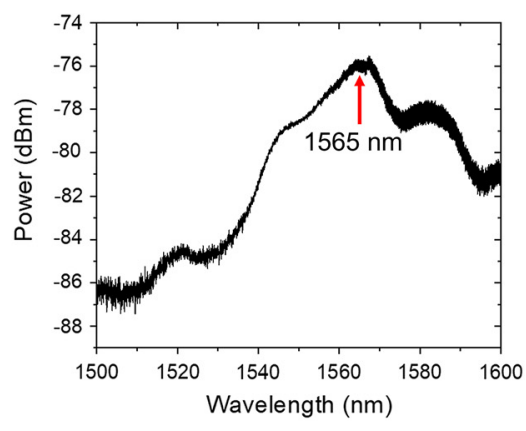

(c)

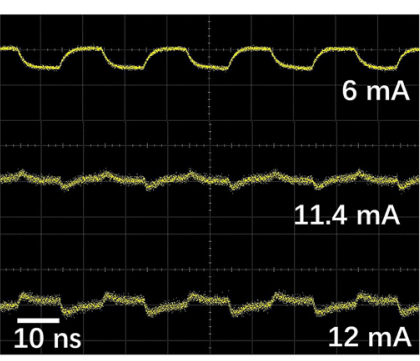

(d)

Fig. 4 (a) Schematics of the experimental setup. (b) Schematics (top view) of the test III-V-on-Si SOA structure. (c) Measured ASE spectrum from the test SOA structure under an injection of $15 \mathrm{~mA}(1.5 \mathrm{~V})$. (d) Measured photocurrent waveforms from the SOA indicating a transparency current of $\sim 11.4 \mathrm{~mA}$. 
We characterize the gain and the transparency current density of the MQW using a test III-Von-Si SOA structure, as shown in Fig. 4(b). We measure the amplified spontaneous emission (ASE) spectrum under an injection of $15 \mathrm{~mA}$. Figure 4(c) shows the measured ASE spectrum with a peak at around $1565 \mathrm{~nm}$. We measure the transparency current density of the MQW based on the method described in Ref. 28. We launch an optical signal at 1560-nm modulated at $100 \mathrm{Mbit} / \mathrm{s}$ into the SOA. We inject different amounts of DC currents into the SOA and read out the photocurrent waveforms from the bias tee by replacing the pattern generator with the oscilloscope. Figure 4(d) shows the measured photocurrent waveforms from this SOA under different injection currents. We observe a nearly flat photocurrent waveform of the modulated incident optical signal under an injection current of $\sim 11.4 \mathrm{~mA}$. Assuming a uniform injection across the SOA, we obtain a transparency current density $J_{\text {tr }}$ of our MQW around $1560 \mathrm{~nm}$ to be $\sim 456 \mathrm{~A} / \mathrm{cm}^{2}$.

\subsection{Below-Threshold: A Gain-Assisted MRR Add-Drop EO Switch}

Figure 5(a) shows the measured through-transmission spectra of the resonances around $1563.7 \mathrm{~nm}$ under different injection currents. Figure 5(b) shows the measured drop-transmission spectra of the resonances around $1563.7 \mathrm{~nm}$ under different injection currents. We obtain all of the spectra using an optical power of $1.7 \mathrm{dBm}$ at the input-coupled fiber tip. We attribute the high insertion loss of the sample to the unoptimized cleaving process, with an estimated $\sim 9 \mathrm{~dB}$ coupling loss at each end of the waveguide.

Figure 5(c) summarizes the measured resonance wavelengths (blue squares) and the loaded quality-factors (Q-factors) (red squares) under different injection currents. The inset shows the measured current-voltage (I-V) curve of the III-V diode. We note the transparency point under an injection current $I_{\mathrm{tr}}=J_{\text {tr }} \times A_{\mathrm{III}-\mathrm{V}} \approx 4 \mathrm{~mA}$ in Fig. 5(c), where the III-V-on-Si MRR behaves essentially as a passive resonator. Figure 5(c) shows a saturated resonance blue-shift of $1.58 \mathrm{~nm}$ as the injection current increases to $8 \mathrm{~mA}$. Further increase of the injection current causes a resonance red-shift, possibly due to the dominance of the TO effect. Meanwhile, the resonance Q-factors of the III-V-on-Si MRR increase as the III-V gain medium increasingly compensates for the cavity optical loss with increasing injection currents.

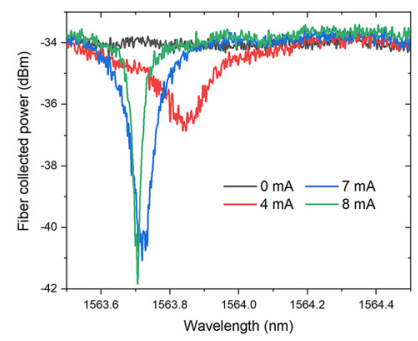

(a)

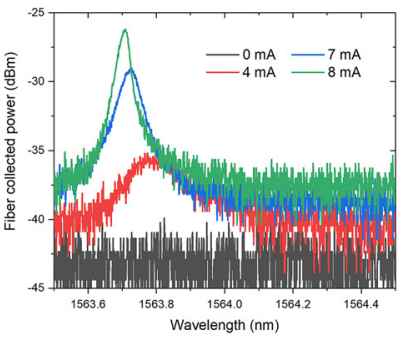

(b)

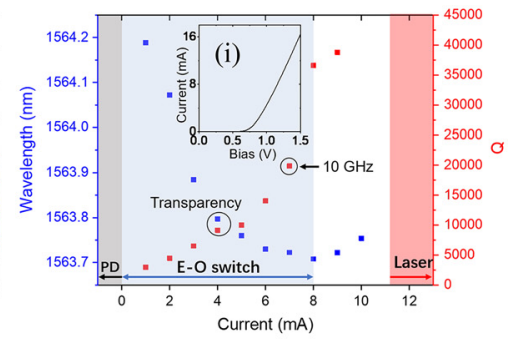

(c)

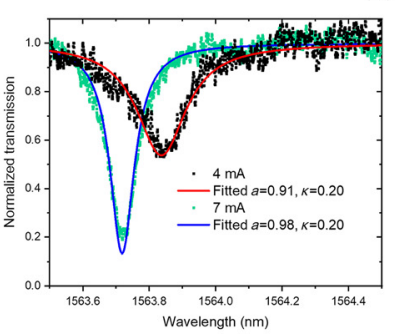

(d)

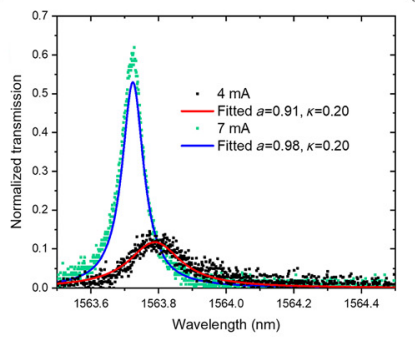

(e)

Fig. 5 (a) and (b) measured through- and drop-port transmission spectra of the resonances around $1563.7 \mathrm{~nm}$ under different injection currents, respectively. (c) Measured resonance wavelength shifts (blue squares) and loaded Q-factors (red squares) over injection currents. Inset (i): Measured current-voltage (I-V) curve. (d) and (e) normalized through- and drop-port transmission spectra and their transfer-matrix model fits under 4 and $7 \mathrm{~mA}$ injection currents, respectively. 
Figures 5(d) and 5(e) show the normalized through- and drop-port transmission spectra and their transfer-matrix model fits under 4 and $7 \mathrm{~mA}$ injection currents, respectively. We subtract the estimated waveguide crossing insertion loss of $\sim 5.8 \mathrm{~dB}$ from the $0 \mathrm{~mA}$ through-port spectrum and treat it as the normalization baseline for the normalization of through-port transmission spectra under other injection currents. This is because under zero injection our device can be treated as a waveguide decoupled from the MRR due to the weak resonance. We perform the fitting using the transfer matrix model described in Ref. 29 to obtain the fitting parameters: (1) the field amplitude cross-coupling coefficient $\kappa$ and (2) the amplitude round trip constant $a\left(a^{2}=e^{-\alpha L}\right)$ of the MRR, where $\alpha\left(\mathrm{cm}^{-1}\right)$ is the power loss (gain) coefficient of the waveguide and $L$ is the cavity round-trip length. We fit the normalized through- and drop-port transmission spectra with the same fitting parameters at transparency $(4 \mathrm{~mA})$. We obtain a relatively small fitted MRR-buswaveguide energy cross-coupling coefficient $\kappa^{2}$ of $\sim 0.04$, possibly caused by fabrication errors in the waveguide widths and coupling gaps.

We operate the switch at the wavelength of the blue-shifted resonance at $1563.72 \mathrm{~nm}$ under $7 \mathrm{~mA}$ injection. We obtain an extinction ratio (ER) between the measured optical power at the drop- and through-ports $\left(P_{d} / P_{t}\right)$ of -10.1 and $11.4 \mathrm{~dB}$ with no injection and with $7-\mathrm{mA}$ injection current, respectively. We also observe a narrowing of the resonance linewidth from $0.7(\sim 87 \mathrm{GHz})$ to $0.08 \mathrm{~nm}(\sim 10 \mathrm{GHz})$ due to partially compensating for the waveguide-coupled cavity loss.

The normalized drop-port transmission spectrum under 7-mA injection current shown in Fig. 5(e) shows a drop-port power transmission of $\sim 0.6$, which is larger than the drop-port power transmission of $\sim 0.15$ under $4-\mathrm{mA}$ transparency injection current. The increased drop-port power transmission indicates a partial compensation of the cavity losses. The fitted amplitude round trip constant $a=0.98$ is very close to unity. This is a workable operational point, as further increase of $a$ would consume more power and possibly begins lasing oscillation. The power consumption for this switch is $7.84 \mathrm{~mW}\left(I_{D C} \times V_{D C}\right)$ under this operation point. The loss compensation capability of our III-V-on-Si MRR optical switch can mitigate the accumulated insertion loss and on-chip signal attenuation, thus enabling them to be cascaded into a large-scale network.

We study the dynamic responses of the single-bus III-V-on-Si MRR. Figure 6(a) shows the measured through-transmission spectra of the III-V-on-Si MRR under different injection currents. The inset illustrates schematically the top-view of the single-bus III-V-on-Si MRR. We obtain a saturated blue-shift of $\sim 1.36 \mathrm{~nm}$ with an injection current of $8 \mathrm{~mA}$. We measure all of the transmission spectra using an optical power of $1.7 \mathrm{dBm}$ at the input-coupled fiber tip.

To perform the switching experiment, we launch a higher optical input power of $15.7 \mathrm{dBm}$ at the fiber tip. Figure 6(b) shows the corresponding through-transmission spectra around $1558 \mathrm{~nm}$. We observe a redshift of the resonance wavelengths as we increase the injection current. We attribute the observed redshift to a higher TO effect induced by the residual absorption of

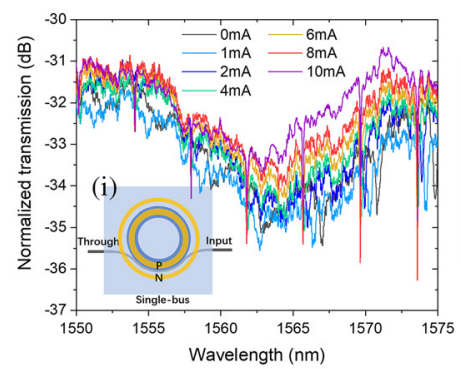

(a)

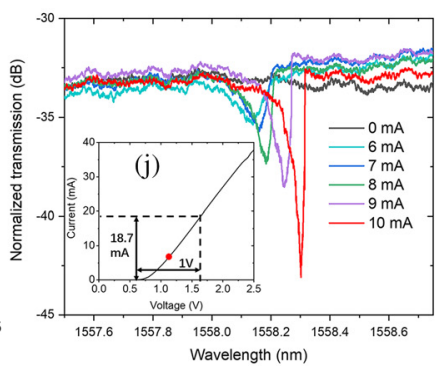

(b)

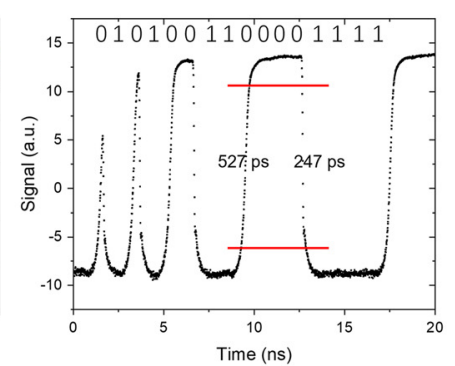

(c)

Fig. 6 (a) Measured through-transmission spectra of the single-bus MRR under different injection currents under an optical input power of $1.7 \mathrm{dBm}$ at the fiber tip. Inset (i): top-view schematics of the single-bus-waveguide MRR. (b) Measured through-transmission spectra around $1558 \mathrm{~nm}$ under different injection currents under an optical input power of $15.7 \mathrm{dBm}$ at the fiber tip. Inset (j): the corresponding I-V curve of this device and the dynamic operation regime. (c) Measured dynamic through-transmission at $1558.28 \mathrm{~nm}$ of the MRR with a square-wave data-modulation between 0.65 and $1.65 \mathrm{~V}$ bias at a speed of $1 \mathrm{Gbit} / \mathrm{s}$. 
the cavity optical power. The DC resonance ER improves from 0 to $10.5 \mathrm{~dB}$ with the injection current increasing from 0 to $10 \mathrm{~mA}$.

We obtain a high-speed on-off switching when we apply a $1 \mathrm{Gbit} / \mathrm{s}$ electrical modulation to the III-V-on-Si MRR. We operate the MRR at a redshifted resonance wavelength of $\sim 1558.28 \mathrm{~nm}$ under a DC injection current of $10 \mathrm{~mA}$. We modulate the bias voltage across the MRR between 0.65 and $1.65 \mathrm{~V}$, corresponding to an injection current between 0 and $18.7 \mathrm{~mA}$, with a fixed data pattern at a speed of $1 \mathrm{Gbit} / \mathrm{s}$. The power consumption for this device is $13 \mathrm{~mW}$. The inset in Fig. 6(b) shows the domain in which we modulate the MRR. Figure 6(c) shows the measured transmission time-trace. We obtain rise/fall times of 247/527 ps, which are comparable to those reported in Refs. 7 and 9. Such an on-off switching speed is applicable for on-chip circuit-switching applications. Further optimizations and a change of the operational mechanism to a possibly quantum-confined Stark effect are required if we further adopt the III-V-on-Si MRR structure as high-speed electro-absorption modulators.

\subsection{Above-Threshold Injection: An On-Chip Directly Modulated Laser Source}

We forward-bias the single-bus-waveguide III-V-on-Si MRR without input-coupling light. By biasing the MRR above the lasing threshold, we can operate the MRR as an on-chip directly modulated laser source for optical signal generation. We observe $\mathrm{CW}$ lasing emission at room temperature $\left(\sim 21.9^{\circ} \mathrm{C}\right)$ when we inject our III-V-on-Si MRR above its lasing threshold current. We collect the emitted light from the single bus-waveguide end-face using a multimode lensed fiber and measure the spectrum using an OSA.

Figure 7(a) shows the measured output light-current (L-I) curve. The L-I curve indicates a lasing threshold current of $\sim 13.9 \mathrm{~mA}$ or a lasing threshold current density of $\sim 1.7 \mathrm{kA} / \mathrm{cm}^{2}$ for the $817 \mu \mathrm{m}^{2}$ III-V MRR, which is comparable to other similar devices on the heterogeneously

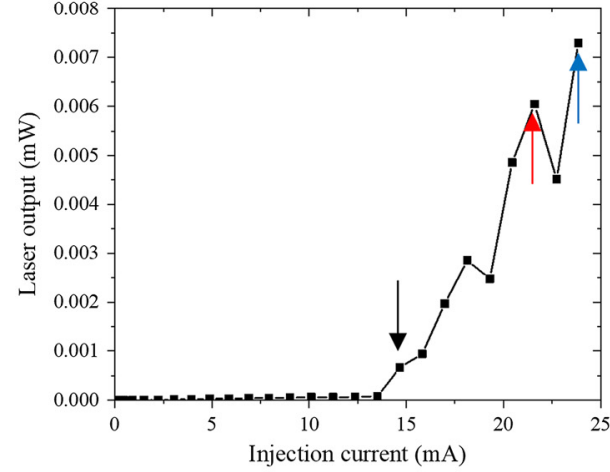

(a)

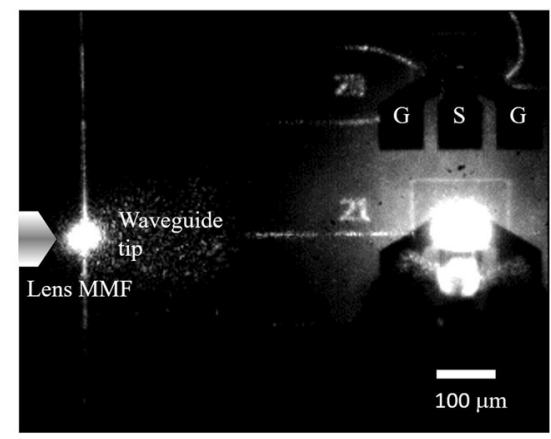

(b)

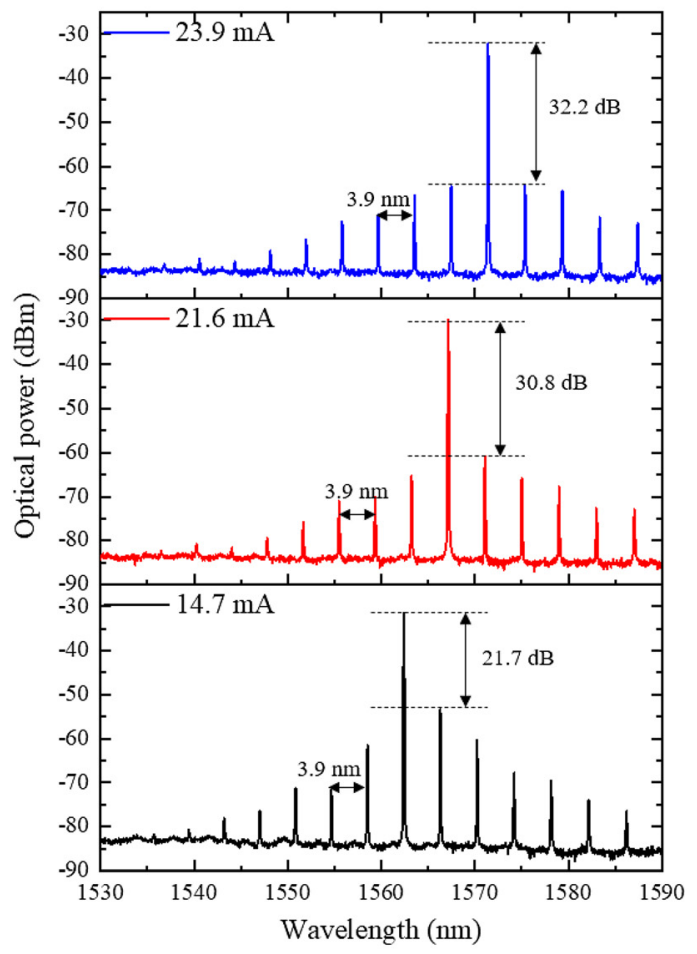

(c)

Fig. 7 (a) Measured L-I curve of an III-V-on-Si MRR at room temperature. The arrowheads label three injection currents with measured lasing emission spectra. (b) Micrograph of the NIR laser emission scattering imaged from the top. (c) Measured lasing spectra collected from the buswaveguide end-face under continuous injection currents of $14.7,21.6$, and $23.9 \mathrm{~mA}$, corresponding to the arrow heads depicted in (a). 


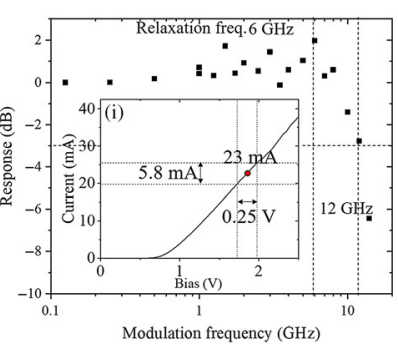

(a)

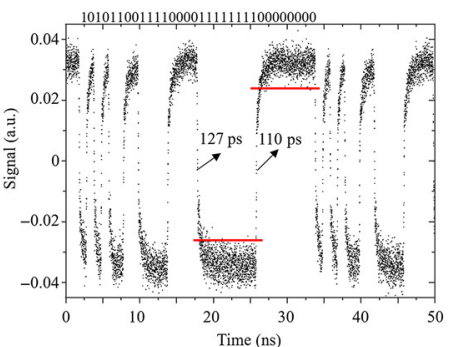

(b)

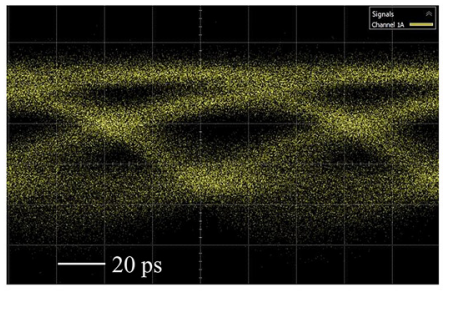

(c)

Fig. 8 (a) Measured small-signal frequency response of the directly modulated laser output. Inset (i): I-V curve of the MRR indicating the operational point and the modulation amplitude for direct modulation of the laser. (b) Measured time trace of the directly modulated laser output with a 32-bit non-return-to-zero data stream at $1 \mathrm{Gbit} / \mathrm{s}$. (c) Measured eye diagram of the directly modulated laser at $10 \mathrm{Gbit} / \mathrm{s}$.

integrated III-V-on-Si platform. ${ }^{30}$ Given a measured coupling loss of $\sim 9 \mathrm{~dB}$ at each facet, we estimate an optical power of $-14 \mathrm{dBm}$ at the waveguide tip when injected with a direct current (20.9 mA) 1.5 times the lasing threshold current. Figure 7(b) shows the measured NIR top-view image of the laser emission scattered from the MRR and the bus-waveguide.

Figure 7(c) shows the measured multimode lasing emission spectra collected at the buswaveguide facet under continuous injection currents of 14.7, 21.6, and $23.9 \mathrm{~mA}$, with the colored arrows in Fig. 7(a) indicating the injection currents on the L-I curve. We observe mode hopping across three different longitudinal modes at different injection currents, consistent with the kinks observed in the L-I curve in Fig. 7(a). We observe a dominant lasing mode at 1562.4, 1567.15, and $1571.4 \mathrm{~nm}$, with side-mode suppression ratios (SMSR) of $\sim 21.7,30.8$, and $32.2 \mathrm{~dB}$ at 14.7, 21.6, and $23.9 \mathrm{~mA}$, respectively. The mode hopping towards longer wavelengths and the overall redshift of the emission peaks suggest a dominance of the TO effect. The mode separations are $\sim 3.9 \mathrm{~nm}$, which are consistent with the free spectral range of the III-V-on-Si MRR. The measured lasing linewidths of $\sim 0.02 \mathrm{~nm}$ are resolution-limited by the OSA.

Table 2 FOM for III-V-on-Si MRRs and state-of-the-art methods.

\begin{tabular}{|c|c|c|c|c|}
\hline & Description & $\begin{array}{l}\text { This work, Add-drop } \\
\text { [single bus, test] }\end{array}$ & $\mathrm{HP}^{33}$ & Ghent $^{9,34}$ \\
\hline \multirow[t]{5}{*}{$\begin{array}{l}\text { Optical switching/ } \\
\text { EO tuning }\end{array}$} & $\begin{array}{l}\text { EO resonance } \\
\text { shift }\left(\delta \lambda_{E O}\right)\end{array}$ & $1.57 \mathrm{~nm}(1.36 \mathrm{~nm})$ & $0.1 \mathrm{~nm}(\mathrm{MOS})$ & $>1 \mathrm{~nm}^{9}$ \\
\hline & $\begin{array}{l}\text { EO tuning injection } \\
\text { current/ bias }\left(I_{o p} / V_{o p}\right)\end{array}$ & $7 \mathrm{~mA}(8 \mathrm{~mA})$ & $5 \mathrm{~V}$ & $55 \mu \mathrm{A}^{9}$ \\
\hline & EO switching fall time $\left(\tau_{R F}\right)$ & NA (527 ps) & NA & $1.2 \mathrm{~ns}^{9}$ \\
\hline & Spectral passband $(\delta \lambda)$ & $\begin{array}{c}0.7 \mathrm{~nm}(87 \mathrm{GHz}, \\
0 \mathrm{~mA}) 0.16 \mathrm{~nm} \\
(19.1 \mathrm{GHz}, 5 \mathrm{~mA}) \\
0.08 \mathrm{~nm}(10 \mathrm{GHz}, \\
7 \mathrm{~mA})\end{array}$ & NA & $\begin{array}{c}0.7 \mathrm{~nm} \\
(87.5 \mathrm{GHz} \\
55 \mu \mathrm{A})^{9}\end{array}$ \\
\hline & Drop-port transmission & $0.6[\mathrm{NA}]$ & NA & $\begin{array}{c}10 \mathrm{~dB} \\
\text { (drop port loss) }^{9}\end{array}$ \\
\hline \multirow[t]{3}{*}{ Lasing } & $\begin{array}{l}\text { Threshold current }\left(I_{t h}\right) \\
\text { (Threshold current } \\
\left.\text { density }\left(J_{t h}\right)\right)\end{array}$ & $\begin{array}{c}11 \mathrm{~mA}\left(1.3 \mathrm{kA} / \mathrm{cm}^{2}\right) \\
{[13.9 \mathrm{~mA}} \\
\left.\left(1.7 \mathrm{kA} / \mathrm{cm}^{2}\right)\right]\end{array}$ & $\begin{array}{c}9-12 \mathrm{~mA} \\
(1.912-2.55 \\
\left.\mathrm{kA} / \mathrm{cm}^{2}\right)\end{array}$ & $350 \mu \mathrm{A}^{34}$ \\
\hline & $\begin{array}{l}\text { Direct modulation } \\
\text { bandwidth }\left(f_{3 \mathrm{~dB}}\right)\end{array}$ & NA (12 Gb/s) & NA & NA \\
\hline & $\begin{array}{l}\text { Energy consumption } \\
\text { per bit }\left(\frac{I_{D C} \times V_{D C}}{\text { datarate }}\right)\end{array}$ & NA (4.3 pJ/bit) & NA & NA \\
\hline
\end{tabular}


Figure 8(a) shows the measured small-signal frequency response of the directly modulated laser as a function of the modulation frequency. The response peaks at a relaxation resonance frequency of $\sim 6 \mathrm{GHz}$. The $3 \mathrm{~dB}$-bandwidth exceeds $12 \mathrm{GHz}$, which compares favorably with other semiconductor MRR lasers. ${ }^{31,32}$ The inset (i) of Fig. 8(a) shows the domain in which we modulate the laser above the threshold, with a modulation amplitude of $5.8 \mathrm{~mA}(250 \mathrm{mV})$ at a DC of $23 \mathrm{~mA}(1.85 \mathrm{~V})$.

Figure 8(b) shows the measured time trace of the directly modulated laser output at $1 \mathrm{Gbit} / \mathrm{s}$ using a 32-bit data stream indicated on top of the graph. The red lines denote the $10 \%$ and $90 \%$ amplitudes. The rise- and fall-times are $\sim 110$ and $\sim 127 \mathrm{ps,}$, respectively. Figure $8(\mathrm{c})$ shows the measured eye diagram of the directly modulated laser upon a pseudo-random bit sequence at $10 \mathrm{Gbit} / \mathrm{s}$, with a modulation amplitude of $250 \mathrm{mV}$. The energy consumption for this direct modulated laser is $4.3 \mathrm{pJ} / \mathrm{bit}\left(\frac{I_{D C} \times V_{D C}}{\text { data rate }}\right)$.

\subsection{Summary of Figures of Merit}

We benchmark the performances of our III-V-on-Si MRRs within different operational regimes with other works with similar structures. The summarized figures of merit (FOMs) are shown in Table 2. The included references are not exhaustive.

\section{Conclusion}

We have demonstrated the proof-of-concept of the multi-functionalities of heterogeneously integrated III-V-on-Si MRRs. Table 2 summarizes the FOM's of our III-V-on-Si MRR device and benchmarks with the state-of-the-art methods using similar structures. We operated the MRR below its lasing threshold as a wavelength-channel EO switch with loss-compensation. We obtained a power-splitting ratio of $11.4 \mathrm{~dB}$ between the drop- and through-ports under an injection current of $7 \mathrm{~mA}$ at a $1.57 \mathrm{~nm}$ blue-shifted resonance wavelength. We obtained a channel switching rise/fall times of the III-V-on-Si MRR of 247/527 ps. As an electrical injection laser, we demonstrated $\mathrm{CW}$ multimode lasing at room temperature, with a lasing threshold current density of $\sim 1.7 \mathrm{kA} / \mathrm{cm}^{2}$ and a side-mode suppression ratio of exceeding $30 \mathrm{~dB}$. We demonstrated the direct modulation of the laser with a $3 \mathrm{~dB}$ bandwidth exceeding $12 \mathrm{GHz}$ and an energy consumption of $4.3 \mathrm{pJ} /$ bit. The III-V-on-Si MRRs can be readily operated as microcavity PDs with zero bias or a reverse bias. The implementation of such heterogeneously integrated MRRs as versatile building blocks for programmable PICs deserve more investigation.

\section{Acknowledgments}

The authors thank the Nanosystem Fabrication Facility (NFF) of the HKUST for device fabrication. This work is supported in part by the Research Grants Council of the Hong Kong Special Administrative Region, China, under Project 16200618 and in part by the GBA Institute of Collaborative Innovation under Project GICI-003. The authors declare no competing financial interests.

\section{References}

1. Z. Yao et al., "Integrated silicon photonic microresonators: emerging technologies," IEEE J. Sel. Top. Quantum Electron. 24(6), 1-24 (2018).

2. Y. Shen et al., "Deep learning with coherent nanophotonic circuits," Nat. Photonics 11(7), 441-446 (2017).

3. S. Keyvaninia et al., "Ultra-thin DVS-BCB adhesive bonding of III-V wafers, dies and multiple dies to a patterned silicon-on-insulator substrate," Opt. Mater. Express 3(1), 35-46 (2013).

4. C.-W. Lee, Y. S. Liang, and D. K.-T. Ng, "Study of ultrathin SiO2 interlayer wafer bonding for heterogeneous III-V/Si photonic integration," Mater. Res. Express 2(9), 096201 (2015). 
Li et al.: Heterogeneously integrated III-V-on-Si microring resonators: a building block...

5. Y. Ikku et al., "ALD Al2O3 activated direct wafer bonding for III-V CMOS photonics platform," in Int. Conf. Indium Phosphide Relat. Mater., IEEE, pp. $24-27$ (2011).

6. M. N. Sysak et al., "Integration of hybrid silicon lasers and electroabsorption modulators," Opt. Express 16(17), 12478-12486 (2008).

7. L. Liu et al., "Carrier-injection-based electro-optic modulator on silicon-on-insulator with a heterogeneously integrated III-V microdisk cavity," Opt. Letters 33(21), 2518-2520 (2008).

8. P. Pintus et al., "Characterization of heterogeneous InP-on-Si optical modulators operating between $77 \mathrm{~K}$ and room temperature," APL Photonics 4, 100805 (2019).

9. L. Liu et al., "Low-power electro-optical switch based on a III-V microdisk cavity on a silicon-on-insulator circuit," Proc. SPIE 7631, 76310P (2009).

10. C. Zhang et al., "Ultralinear heterogeneously integrated ring-assisted Mach-Zehnder interferometer modulator on silicon," Optica 3(12), 1483 (2016).

11. M. L. Davenport, S. Liu, and J. E. Bowers, "Integrated heterogeneous silicon/III-V modelocked lasers," Photonics Res. 6(5), 468 (2018).

12. Y. Hu et al., "III/V-on-Si MQW lasers by using a novel photonic integration method of regrowth on a bonding template," Light Sci. Appl. 8, 93 (2019).

13. K. Van Gasse, R. Wang, and G. Roelkens, " $27 \mathrm{~dB}$ gain III-V-on-silicon semiconductor optical amplifier with $>17 \mathrm{dBm}$ output power," Opt. Express 27(1), 293 (2019).

14. M. L. Davenport et al., "Heterogeneous silicon/III-V semiconductor optical amplifiers," IEEE J. Sel. Top. Quantum Electron 22(6), 78-88 (2016).

15. C. Zhang et al., " $8 \times 8 \times 40$ Gbps fully integrated silicon photonic network on chip," Optica 3(7), 785 (2016).

16. J. C. Hulme et al., "Fully integrated hybrid silicon two dimensional beam scanner," Opt. Express 23(5), 5861 (2015).

17. A. N. Tait et al., "Neuromorphic photonic networks using silicon photonic weight banks," Sci. Rep. 7(1), 7430 (2017).

18. D. A. B. Miller, "Self-configuring universal linear optical component [Invited]," Photonics Res. 1(1), 1 (2013).

19. D. Pérez, I. Gasulla, and J. Capmany, "Field-programmable photonic arrays," Opt. Express 26(21), 27265 (2018).

20. L. Zhuang et al., "Programmable photonic signal processor chip for radiofrequency applications," Optica 2, 854-859 (2015).

21. R. L. Espinola et al., "Fast and low-power thermooptic switch on thin silicon-on-insulator," IEEE Photonics Technol. Lett. 15(10), 1366-1368 (2003).

22. B. R. Bennett, R. A. Soref, and J. A. Del Alamo, "Carrier-induced change in refractive index of InP, GaAs, and InGaAsP," IEEE J. Quantum Electron. 26(1), 113-122 (1990).

23. H. Park et al., "Hybrid silicon evanescent laser fabricated with a silicon waveguide and III-V offset quantum wells," Opt. Express 13(23), 9460-9464 (2005).

24. T. Fujii et al., "Development of an epitaxial growth technique using III-V on a Si platform for heterogeneous integration of membrane photonic devices on Si," Appl. Sci. 11, 1801 (2021).

25. J. Kim and T. W. Kim, "Initial surface reactions of atomic layer deposition," J. Miner. Met. Mater. Soc. 61, 17-22 (2009).

26. R. L. Puurunen and W. Vandervorst, "Island growth in the atomic layer deposition of zirconium oxide and aluminum oxide on hydrogen-terminated silicon: Growth mode modeling and transmission electron microscopy," J. Appl. Phys. 96(9), 4878-4889 (2004).

27. Y. Zheng et al., "High-quality-factor AlGaAs-on-sapphire," J. Lightwave Technol. 37(3), 868-874 (2019).

28. L. F. Tiemeijer et al., "Direct measurement of the transparency current and valence band effective masses in tensile and compressively strained $\mathrm{InGaAs} / \mathrm{InP}$ multiple quantum-well laser amplifiers," Appl. Phys. Lett. 60(5), 554-556 (1992).

29. W. Bogaerts et al., "Silicon microring resonators," Laser Photonics Rev. 6(1), 47-73 (2012).

30. D. Liang et al., "Low threshold electrically-pumped hybrid silicon microring lasers," IEEE J. Sel. Top. Quantum Electron. 17(6), 1528-1533 (2011).

31. A. Kapsalis et al., "7 Gb/s direct modulation of vertically coupled microring lasers," in Conf. Opt. Fiber Commun./Natl. Fiber Opt. Eng. Conf., Vols. 1-8, pp. 2917-2919 (2008). 
32. A. Bennecer et al., "Directly modulated wavelength-multiplexed integrated microring laser array," IEEE Photonics Technol. Lett. 20, 1411-1413 (2008).

33. D. Liang et al., "Fully-integrated heterogeneous DML transmitters for high-performance computing," J. Lightwave Technol. 38(13), 3322-3337 (2020).

34. T. Spuesens et al., "Improved design of an InP-based microdisk laser heterogeneously integrated with SOI," in 6th IEEE Int. Conf. Group IV Photonics (2009).

Jiayang $\mathbf{L i}$ is a postgraduate student at the Hong Kong University of Science and Technology. He received his BS degree in optoelectronic science and technology from Huazhong University of Science and Technology, Wuhan, China, in 2018. His current research interests include heterogeneously integrated III-V-on-silicon photonics and nonlinear optics.

Bo Xue Tan is a postgraduate student at the Hong Kong University of Science and Technology. He received his BS degree in physics from National University of Singapore, Singapore, in 2016. His current research interests include heterogeneously integrated III-V-on-silicon photonics and nonlinear optics.

Mingle Tian is a postgraduate student at the Hong Kong University of Science and Technology. He received his BS degree in Electronic Engineering from City University of Hong Kong, Hong Kong, in 2019. His current research interests include heterogeneously integrated III-V-on-silicon photonics and neuromorphic computing.

Andrew W. Poon is a professor at The Hong Kong University of Science and Technology. He received his BA degree in physics from the University of Chicago, Illinois, in 1995 and his MPhil and PhD in physics from Yale University, New Haven, Connecticut, in 1998 and 2001, respectively. His current research interests include silicon photonics, heterogeneously integrated III-V-on-silicon photonics, optical microresonators, on-chip optical interconnects, integrated nonlinear and quantum photonics, optical manipulation of particles/cells, and biosensing. 